\title{
Two-dimensional excitonic emission in InAs submonolayers
}

\author{
Z. L. Yuan, ${ }^{*}$ Z. Y. Xu, ${ }^{*}$ B. Z. Zheng, J. Z. Xu, and S. S. Li* \\ National Laboratory for Superlattices \& Microstructures, Institute of Semiconductors, Chinese Academy of Sciences, \\ P.O. Box 912, Beijing 100083, China \\ Weikun Ge, Y. Wang, J. Wang, and L. L. Chang \\ Department of Physics, Hong Kong University of Science \& Technology, Clear Water Bay, Kowloon, Hong Kong \\ P. D. Wang ${ }^{\dagger}$ and C. M. Sotomayor Torres \\ Nanoelectronics Research Centre, Department of Electronics and Electrical Engineering, University of Glasgow, \\ Glasgow G12 8QQ, United Kingdom \\ N. N. Ledentsov \\ A. F. Ioffe Physico-Technical Institute, 26 Politekhnicheskaya Street, St. Petersburg 198904, Russia
}

(Received 22 January 1996)

\begin{abstract}
Photoluminescence (PL) and time-resolved photoluminescence (TRPL) were used to study optical emissions of ultrathin InAs layers with average layer thickness ranging from $\frac{1}{12}$ to $1 \mathrm{ML}$ grown on GaAs substrates. We have found that the inhomogeneous broadening of the PL from InAs layers can be well described by the quantum-well model with InAs islands coupling to each other and being regarded as a quasiwell. From the temperature dependence of the exciton linewidth, the exciton-LO-phonon scattering coefficient was found to be comparable to that in conventional two-dimensional quantum wells. In the TRPL measurements, the PL decay time increases linearly with temperature, which is a typical characteristic of free excitons in quantum wells. All these results indicate that the excitons localized in InAs exhibit two-dimensional properties of quantum wells, despite the topographical islandlike structure. [S0163-1829(96)01647-5]
\end{abstract}

\section{INTRODUCTION}

Recently much interest has focused on the growth of highly strained $\mathrm{In}(\mathrm{Ga}) \mathrm{As}-\mathrm{GaAs}$ heterostructures on GaAs substrates ${ }^{1-10}$ because such a strained epitaxy may provide a direct means to form quantum wires or dots without any patterning process. It has been reported that the growth of highly strained InAs layers onto GaAs substrates could lead to spontaneous formations of clusters, so-called selforganized quantum dots. ${ }^{3}$

More recently, Wang et al. ${ }^{7}$ found that a uniform array of InAs wires elongated along the [01 $\overline{1}]$ direction is formed in the case of submonolayer (SML) InAs deposition on GaAs substrates from an optical anisotropy study. Scanningtunneling-microscopy studies have revealed that the growth of InAs SML structure on (100) or vicinal GaAs surfaces results in a spontaneous formation of coherent InAs ML-high wirelike islands with a width of $\sim 4 \mathrm{~nm}$ and an elongation along the $[01 \overline{1}]$ direction. ${ }^{8}$ Transmission-electronmicroscopy (TEM) studies also displayed the coherent islands with a typical lateral size of $5 \mathrm{~nm}$ in an InAs SML deposited on (100) GaAs. ${ }^{9}$ Many optical studies on an InAs SML structure demonstrated its high luminescence efficiency, ${ }^{7}$ large exciton oscillator strength, ${ }^{10}$ and huge binding energy. ${ }^{11}$ The above phenomena were interpreted in terms of the lateral quantum confinement. On the other hand, Brandt, Lage, and Ploog ${ }^{12}$ found that excitons localized in an ultrathin (0.8-ML) InAs layer still show two-dimensional properties in optical response experiments. Clearly, the optical properties of such a system still remain poorly under- stood. Does an exciton in such structures exhibit zerodimensional (0D) properties? Does an exciton feel the confinement of the isolated islands when their lateral size (approximately $50 \AA$ ) (Ref. 9) is smaller than the exciton diameter $(250 \AA)$ ?

In this paper, we used temperature-dependent continuouswave $(\mathrm{cw})$ and time-resolved photoluminescence (PL) to study the optical properties of SML InAs structures embedded in a GaAs matrix. Based on the temperature-dependent PL linewidth analysis, the exciton-LO-phonon scattering coefficient was estimated to be $12 \mathrm{meV}$, which is comparable to that of typical quantum wells. In time-resolved photoluminescence (TRPL) measurements, the measured PL decay time is a strong function of the average InAs thickness, from $2.1 \mathrm{~ns}$ for $\frac{1}{12} \mathrm{ML}$ to $0.35 \mathrm{~ns}$ for $1 \mathrm{ML}$. This result is similar to that obtained in very thin quantum wells (QW's), which was attributed to the penetration of the envelope wave function of electrons into the barriers. In addition, the PL decay time increases linearly with temperature, showing a typical characteristic of free excitons in quantum wells. From our experiments we conclude that the exciton emission in InAs SML structures is of two-dimensional (2D) nature. The structure can be treated as a very thin quantum well, and the InAs island distribution as interface roughness.

\section{EXPERIMENTAL DETAILS}

The structures were grown by elemental source molecular-beam epitaxy on (100) GaAs semi-insulating substrates. After oxide desorption a $0.3-\mu$ m-thick GaAs buffer 


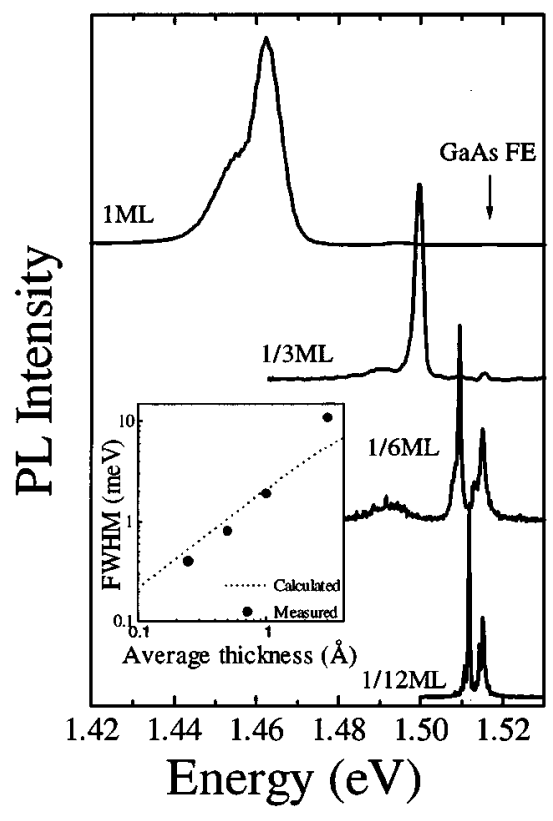

FIG. 1. PL spectra of four samples at $10 \mathrm{~K}$. The excitation power was selected at a very low level of $0.1 \mathrm{~mW}$. The inset depicts the calculated (dashed line) and measured exciton linewidth dependencies on the average InAs layer thickness.

layer was grown at $600{ }^{\circ} \mathrm{C}$. This layer was followed by a 40-period 25 - $\AA$ GaAs/25- $\AA \mathrm{Al}_{0.4} \mathrm{Ga}_{0.6}$ As superlattice (SL) to trap impurities and to prevent nonequilibrium carrier spreading into the semi-insulating substrate. The SL was followed by $2000-\AA \mathrm{GaAs}$, then the substrate temperature was lowered to $450{ }^{\circ} \mathrm{C}$, and the arsenic beam equivalent pressure was increased to $10^{-5}$ Torr. Subsequently, a $100-\AA$ GaAs layer was deposited, which was followed by a single InAs layer, and $20 \AA$ of GaAs was grown on top. The substrate temperature was then increased to $600{ }^{\circ} \mathrm{C}$, with the arsenic pressure reduced to $3 \times 10^{-6}$ Torr. Under this condition, a $2000-\AA$ thick GaAs layer and a 40-period 25- $\AA$ GaAs/25- $\AA$ $\mathrm{Al}_{0.4} \mathrm{Ga}_{0.6} \mathrm{As}$. Superlattices were grown to prevent surface recombination and to avoid surface-related electric fields. Finally, a $100-\AA \mathrm{GaAs}$ cap layer was grown to protect the surface. The details of the growth were published elsewhere. ${ }^{7}$

For TRPL measurements, the excitation of the samples was carried out with a dicyanomethylene (DCM) dye laser synchronously pumped by a mode-locked $\mathrm{Ar}^{+}$laser with a repetition rate of $82 \mathrm{MH}_{z}$. The $\mathrm{PL}$ signal was dispersed by a small monochromator and detected, by a 2D synchroscan streak camera with a total time resolution of $30 \mathrm{ps}$. For cw PL measurements, the dye laser worked in a continuouswave mode, and the signal from the samples was dispersed by a SPEX $1403(0.85 \mathrm{~m})$ spectrometer with an energy resolution of $0.1 \mathrm{meV}$, and detected by a cooled GaAs photomultiplier and a photon counting system.

\section{RESULTS AND DISCUSSIONS}

\section{A. cw PL measurements}

Figure 1 shows low-temperature PL spectra of structures containing InAs layers with different average InAs layer
TABLE I. Average thickness of SML InAs samples, and the exciton energy and FWHM obtained from PL spectra at $10 \mathrm{~K}$.

\begin{tabular}{lccc}
\hline \hline Sample & Average $L_{Z}(\mathrm{ML})$ & $E_{\mathrm{hh}}(\mathrm{eV})$ & FWHM $(\mathrm{meV})$ \\
\hline $\mathrm{A}$ & 1 & 1.4635 & 9.5 \\
$\mathrm{~B}$ & $1 / 3$ & 1.5000 & 1.9 \\
$\mathrm{C}$ & $1 / 6$ & 1.5097 & 0.8 \\
$\mathrm{D}$ & $1 / 12$ & 1.5118 & 0.4 \\
\hline \hline
\end{tabular}

thickness of $\frac{1}{12}, \frac{1}{6}, \frac{1}{3}$, and $1 \mathrm{ML}(1 \mathrm{ML}=3.00 \AA)$, respectively. Both InAs-related exciton transitions and GaAs free-exciton (FE) emissions $(1.515 \mathrm{eV})$ are observed in all four samples. Three remarkable features in the spectra are that (i) the full width at half maximum (FWHM) of the InAs heavy-hole (hh) exciton line decreases with decreasing InAs thickness, from $9.5 \mathrm{meV}$ for $1 \mathrm{ML}$ to $0.4 \mathrm{meV}$ for $\frac{1}{12} \mathrm{ML}$; (ii) the InAs-related emission energy increases with the decrease of the average thickness of the InAs layer; (iii) the PL intensity ratio between the InAs-related emission energy and that from GaAs emissions decreases with decreasing InAs thickness. In Table I we list sample parameters, and the exciton energies and linewidths obtained from a PL measurement at $10 \mathrm{~K}$.

The exciton line broadening in semiconductor quantum wells is related to various scattering processes, and to an inhomogeneous fluctuation of the well thickness. At low temperatures the exciton linewidth is expressed as

$$
\Gamma=\Gamma_{0}+\Gamma_{\text {in }},
$$

where $\Gamma_{0}$ is the homogeneous linewidth of the exciton line, mainly due to acoustical-phonon interaction and spin-flip scattering, ${ }^{13}$ and $\Gamma_{\text {in }}$ is the inhomogeneous spectral broadening mainly originated from interface roughness. According to the virtual-crystal approximation (VCA) model proposed by Singh and co-workers, ${ }^{14}$ the exciton cannot "feel", the individual single growth islands when the lateral size of such islands is smaller than the exciton Bohr radius $\left(a_{B}\right)$. The island distribution only contributes to the spectral broadening $\Gamma_{\text {in }}$ of the exciton emission, which can be written as ${ }^{15}$

$$
\begin{aligned}
& \Gamma_{\text {in }}=\left.\frac{\partial E_{g}^{2 \mathrm{D}}}{\partial L_{Z}}\right|_{L_{Z}=\left\langle L_{Z}\right\rangle} \delta_{s}, \\
& \delta_{s}=\delta \sqrt{p(1-p)} \frac{d_{I}}{2 a_{B}},
\end{aligned}
$$

where $d_{I}$ is the average lateral extension of interface islands of height $\delta, a_{B}$ the Bohr radius of the exciton, $p(0<p<1)$ the average island coverage of the heterointerface, and $\left\langle L_{Z}\right\rangle$ the mean QW thickness, while $\delta_{s}$ represents the standard deviation.

Using this quantum-well model we fitted the exciton linewidth dependence on the InAs layer thickness $L_{Z}$ under the assumptions that (i) the InAs layers are treated as very thin quantum wells, and (ii) the InAs island structure is regarded as interface roughness which inhomogeneously broadens the exciton linewidth. In the inset of Fig. 1 we show the best fit to the exciton linewidth dependence on the average InAs layer thickness, together with the experimental data (solid circles). In the calculation the effective-mass approximation 


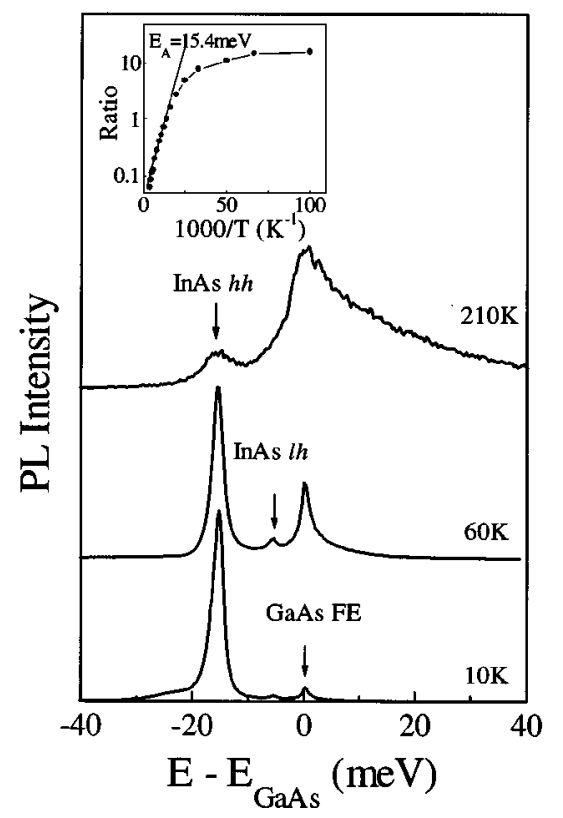

FIG. 2. PL spectra measured at several temperatures for sample $B$ under the excitation power of $1 \mathrm{~mW}$. The inset shows the Arrhenius plot of the PL intensity ratio between the InAs hh and GaAs FE.

(EMA) was used, and the well width was replaced by the average InAs thickness. ${ }^{16}$ For simplicity the same standard deviations $\delta_{s}$ were used for all samples. ${ }^{17}$ It can be seen from the inset of Fig. 1 that the calculated FWHM's are in good agreement with the measured ones, indicating that the quantum-well model is applicable to the InAs SML structures. In other words the InAs SML can indeed be treated as a conventional quantum well with its interface roughness determined by the average InAs coverage. From the line broadening, we may estimate the lateral size of the growth islands. Assuming the exciton Bohr radius to be $150 \AA$, the value often used in $2 \mathrm{D}$ quantum wells, for $\left\langle L_{Z}\right\rangle=\frac{1}{3} \mathrm{ML}(p=$ $\left.\frac{1}{3}\right)$ the estimated lateral size $d_{I}$ of the 1-ML-high ( $\left.\delta=1 \mathrm{ML}\right)$ islands $\mathrm{s}^{7-9}$ is about $35 \AA$, which is in good agreement with the TEM results observed by Alonso et al. ${ }^{9}$

PL spectra measured at several temperatures are shown in Fig. 2 for sample B. The InAs hh exciton dominates the entire luminescence processes at low temperatures. As temperature increases, the intensity of the InAs luminescence becomes weaker. At high temperatures, the GaAs freeexciton recombinations gradually dominate the luminescence processes. This is caused by the thermal activation of InAs excitons back to GaAs, where they recombine through radiative and nonradiative channels. The inset shows the Arrhenious plot of the intensity ratio between the InAs- and GaAsintegrated luminescence intensities. From the curve we can obtain the activation energy $E_{A}$. It is very interesting that $E_{A}$ exactly equals the confinement energy. This indicates the high quality of the investigated samples. ${ }^{18}$ For a 1-ML sample, similar results are obtained (not shown here). Another remarkable feature in Fig. 2 is that, as temperature increases, the luminescence line is broadened, especially in the high-temperature regime. Figure 3 shows the InAs hh exciton linewidth as a function of temperature. [Note that the linewidth represents the half-width at half maximum

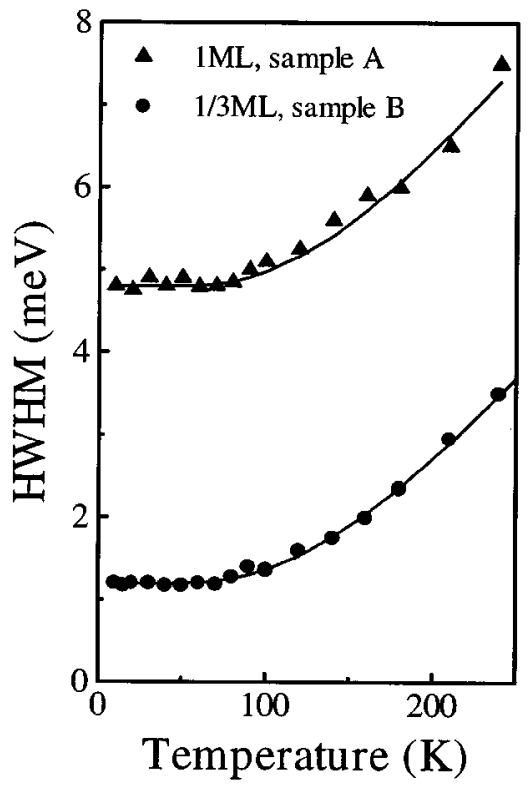

FIG. 3. Temperature dependencies of the FWHM for samples A and B. The solid lines are the best fit to Eq. (3).

(HWHM) here. At high temperatures we determined the HWHM from the low-energy side of the spectra to avoid the influence of thermal population effect of high-energy levels and the interference with the GaAs luminescence.]

In the following we further explore the two-dimensional nature of excitons in SML InAs through the temperature dependence of the exciton linewidth, which reflects the exciton-phonon scattering coefficients. ${ }^{19-23}$ As the acousticphonon broadening is very small compared with the LOphonon broadening, a typical expression for the temperaturedependent linewidth $\Gamma(T)$ can be written ${ }^{19,20}$

$$
\Gamma(T)=\Gamma(0)+\Gamma_{\mathrm{LO}} /\left[\exp \left(E_{\mathrm{LO}} / k_{B} T\right)-1\right]
$$

where $\Gamma(0)$ is the inhomogeneous broadening at zero temperature, and $E_{\mathrm{LO}}$ is the LO-phonon energy. $\Gamma_{\mathrm{LO}}$ is the LOphonon-scattering coefficient, proportional to the sum of possibilities of an exciton scattered from the ground state to all possible states by LO phonons. Apparently, the second term in Eq. (3) represents broadening due to LO-phonon anti-Stokes scattering.

Equation (3) is valid for all dimensionalities. The difference among different dimensionalities lies in the value of the scattering coefficient. When a system's dimensionality approaches zero, the electron density of states is no longer continuous. Difficulty should then appear in the exciton-LOphonon-scattering processes because of the requirement of energy conservation in scattering processes. ${ }^{24,25}$ Therefore, in the zero-dimensional system, no significant thermal broadening effect would be expected. ${ }^{4}$

The data in Fig. 3 can be well fitted using Eq. (3) with $E_{\mathrm{LO}}=36 \mathrm{meV}$ of the GaAs LO-phonon energy. Note that here the LO phonons are lattice vibrations of the GaAs matrix, indicating that InAs embedded in GaAs is similar to impurities in GaAs. The scattering coefficient $\Gamma_{\mathrm{LO}}$ was found to be $\sim 12 \pm 1 \mathrm{meV}$ for both samples. Such a value is much smaller compared with that of GaAs bulk material $[20 \mathrm{meV}$ 


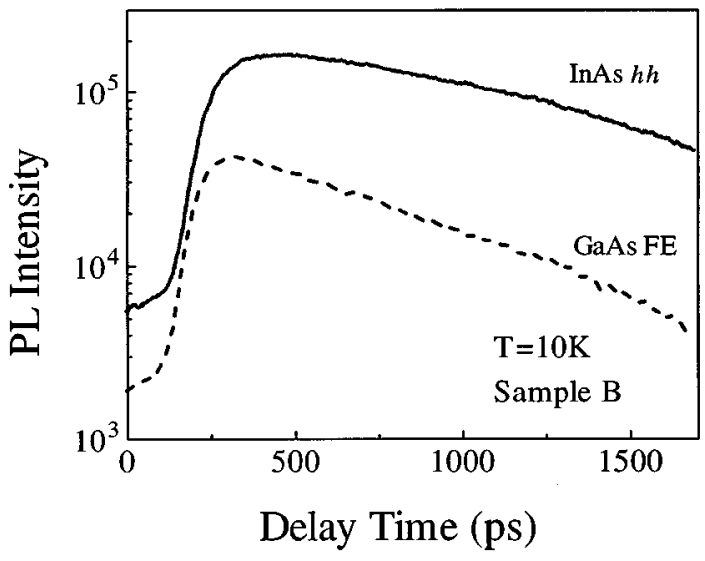

FIG. 4. Temporal evolution of GaAs and InAs exciton luminescence for SML sample B.

(Ref. 20)], indicating confinement in the growth direction. Also, it is very close to that found in GaAs/ $/ \mathrm{Al}_{x} \mathrm{Ga}_{1-x} \mathrm{As}$ (Refs. 21 and 22) and $\operatorname{In}_{x} \mathrm{Ga}_{1-x} \mathrm{As} / \mathrm{GaAs}$ (Ref. 23) quantum wells, demonstrating that scatterings between carriers and LO phonons are still efficient and that carriers in InAs SML structures have two-dimensional properties. ${ }^{16}$ In other words, the LO-phonon-scattering rate is not reduced, though InAs has an island distribution in the GaAs matrix ${ }^{7-9}$ i.e., there is no phonon bottleneck effect. This high LO-phononscattering rate accounts for high luminescence efficiencies in SML structures. The fast scattering rate is further confirmed in the study of hot-carrier relaxation in InAs SML structures. $^{26}$

\section{B. Time-resolved PL measurements}

The decay process of exciton emission in an InAs SML has been studied using time-resolved PL spectroscopy. Figure 4 shows the typical temporal profiles of GaAs and InAs luminescence at $10 \mathrm{~K}$ for the $\frac{1}{3}$-ML sample. The intensity of the InAs luminescence decays monoexponentially after an approximate $250-\mathrm{ps}$ rising time. The relative delay $(\sim 80 \mathrm{ps})$ of the InAs luminescence with respect to the GaAs luminescence is believed to be associated with the carrier trapping process from the GaAs barrier to the InAs islands. The high efficiency of the luminescence indicates efficient trapping and relaxation processes regardless of the islandlike structure. Moreover, the measured 250-ps rise time is comparable to that found in typical 2D quantum wells, ${ }^{27}$ suggesting again that the phonon bottleneck effect is not dominant in our InAs structure. In addition, the high efficiency of the luminescence, the narrow linewidth, and the monoexponential decay indicate that the emission processes are dominated by excitonic recombinations. ${ }^{16}$ The exciton radiative lifetime could thus be directly obtained from the exponential decay curves. In Fig. 5 we plotted the PL decay time $\tau$ of the InAs hh exciton emission as a function of the InAs average thickness at $10 \mathrm{~K}$. An increasing decay time with decreasing InAs thickness was observed, and a very long PL decay time (2.1 ns) was measured from the sample of $\frac{1}{12} \mathrm{ML}$.

It is generally known that the exciton lifetime is inversely proportional to its coherence volume over which the coherent polarization of the exciton persists. ${ }^{28}$ The lifetime in

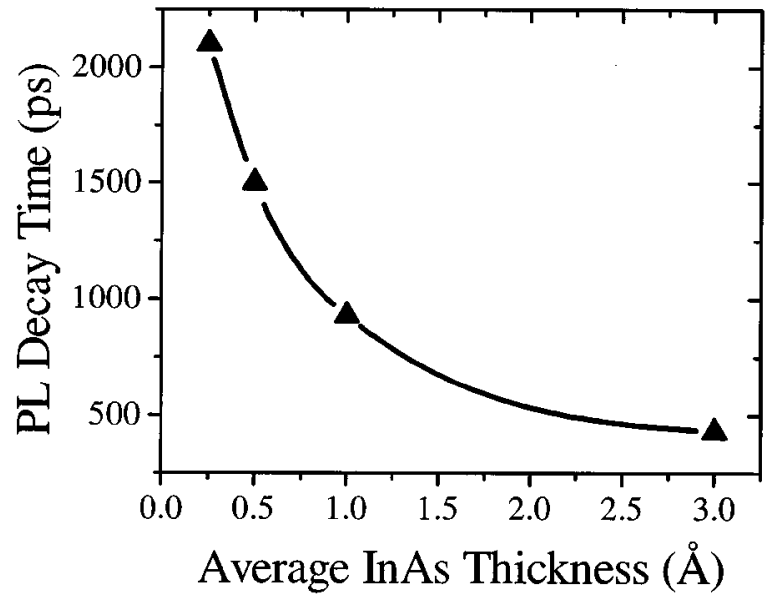

FIG. 5. Well-width dependence of the PL decay time.

quantum-dot or quantum-wire systems is expected to be longer than that in QW's because of the reduction of the coherence volume. ${ }^{29}$ However, this is only valid for the coherent volume being larger than the exciton diameter, ${ }^{30}$ and is not effective to describe the exciton dynamics in InAs SML structures where the lateral extension of the growth islands is much smaller than the exciton diameter. We found that the recombination process in our InAs SML can be understood in the framework of thin quantum wells. As reported for very narrow QW's, ${ }^{31}$ the increasing PL decay time with decreasing InAs thickness can be attributed to penetration of the electron envelope wave function into the GaAs barriers. In the limit of thickness approaching zero, the quasi-2D excitons become quasi-3D excitons of the barrier material. ${ }^{31}$ As shown in Fig. 5, the measured decay times are $2.1,1.5,0.8$, and $0.3 \mathrm{~ns}$ for InAs average thicknesses of $\frac{1}{12}$, $\frac{1}{6}, \frac{1}{3}$, and $1 \mathrm{ML}$, respectively. It is very clear that the exciton lifetime approaches the bulk GaAs value ${ }^{32}(10 \mathrm{~ns}$ at $10 \mathrm{~K})$ as the average InAs thickness decreases. In addition, the increasing lifetime in the SML with thinner InAs average thickness is responsible for the decreasing relative PL intensity of InAs hh exciton emission, as seen in Fig. 1.

To explore the carrier dynamics in InAs SML structures further, we also investigated the temperature dependence of the excitonic lifetime (Fig. 6). At low temperatures both curves exhibit a flat temperature dependence. This is believed to be associated with the exciton localization effect at low temperatures. ${ }^{33}$ At higher temperatures however, the PL decay times present a linear temperature dependence for both samples, that is a typical characteristic of free excitons in quantum wells. ${ }^{28}$ In QW's, only excitons with small momentum $(\mathbf{k} \approx \mathbf{0})$ can recombine radiatively because of the requirement of momentum conservation. When temperature increases, an increased number of excitons will populate states with large $\mathbf{k}$ which cannot recombine radiatively, leading to an increase of the exciton lifetime. Due to the properties of the 2D density of states in QW's, it is expected that the lifetime of excitons increases linearly with temperature. ${ }^{28,34}$ In our cases, localized excitons dominate the luminescence processes at low temperatures. As the temperature increases, localized excitons are thermally-activated to form free excitons (extended states) which have a dispersion in $\mathbf{k}$ space. So the excitonic lifetime increases linearly with temperature in 


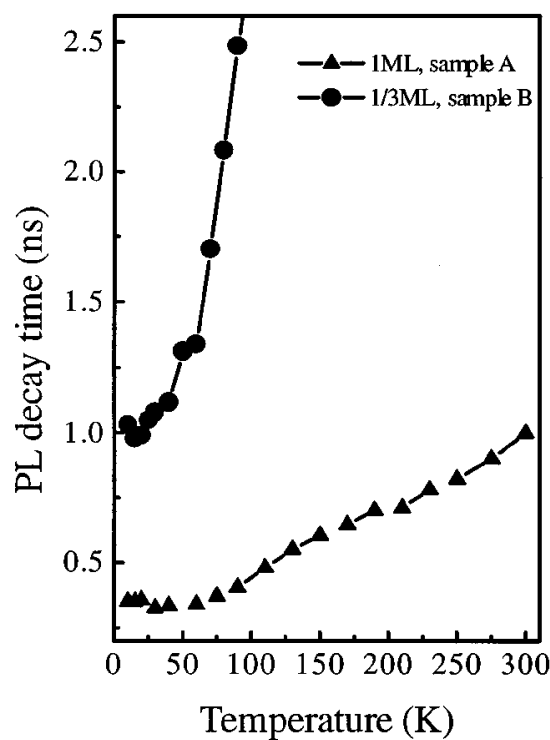

FIG. 6. Temperature dependencies of the PL decay time for samples $\mathrm{A}$ and $\mathrm{B}$.

the high-temperature range. We therefore conclude that the InAs SML structure exhibits 2D properties in its exciton dynamics. On the other hand, in the case of a $0 \mathrm{D}$ system, there is no dispersion in $\mathbf{k}$ space, and no $\mathbf{k}$-conservation requirement for excitonic radiative recombination. The exciton lifetime in this case would depend only on its coherent length, and have no dependence on temperature, contrary to our observation. We may then safely suggest the InAs insertion in GaAs only changes the center-of-mass motion of the exciton in the growth direction, but does not confine the center-ofmass motion of the exciton along the plane at high temperatures. The SML plane acts just like a QW with great interface roughness, and the $2 \mathrm{D}$ free-exciton recombination dominates the recombination processes at high temperatures.

\section{CONCLUSION}

In this paper, we examined the optical behavior of SML InAs structures embedded in a GaAs matrix by using PL and TRPL experiments. It was found that the PL linewidth decreases with the average InAs thickness, and can be described in the framework of the quantum-well model. The temperature-dependent linewidth analysis shows that carriers confined in a SML InAs insertion have a large exciton-LOphonon-scattering coefficient. TRPL measurements revealed that the exciton lifetime increases linearly with temperature. All these results indicate that excitons confined in SML InAs have two-dimensional properties, and the distribution of islandlike InAs in the InAs plane does not prohibit exciton center-of-mass motion in lateral direction.

\section{ACKNOWLEDGMENTS}

The authors would like to thank Professor D. S. Jiang for helpful discussions. The work at Beijing was supported by the National Natural Science Foundation of China and the State Key Program for Basic Research, and the work at The Hong Kong University of Science and Technology (HKUST) was supported by Grant MFG94/95.SC03. Z.L.Y. and Z.Y.X. are grateful to the Department of Physics of HKUST for financial support during their visit in Hong Kong.
*Also at the Department of Physics, Hong Kong University of Science and Technology, Clear Water Bay, Kowloon, Hong Kong.

†Present address: Department of Electrical Engineering, University of Norte Dame, Norte Dame, IN 46556-5637.

${ }^{1}$ J. Y. Marzin and J. M. Gerard, Phys. Rev. Lett. 62, 2172 (1989).

${ }^{2}$ O. Brandt, L. Tapfer, R. Cingolani, K. Ploog, M. Hohenstein, and F. Phillipp, Phys. Rev. B 41, 12599 (1990).

${ }^{3}$ D. Leonard, M. Krishnamurthy, C. M. Reaves, S. P. Denbaars, and P. M. Petroff, Appl. Phys. Lett. 63, 3203 (1993).

${ }^{4}$ M. Grundmann, J. Christen, N. N. Ledentsov, J. Böhrer, D. Bimberg, S. S. Rumimov, P. Werner, U. Richter, U. Gösele, J. Heydenreich, V. M. Ustinov, A. Yu. Egorov, A. E. Zhukov, P. S. Kop'ev, and Zh. I. Alferov, Phys. Rev. Lett. 74, 4043 (1995).

${ }^{5}$ J. M. Moison, F. Houzay, F. Barthe, L. Leprince, E. André, and O. Vatel, Appl. Phys. Lett. 64, 196 (1994).

${ }^{6}$ G. H. Li, A. R. Goñi, K. Syassen, O. Brandt, and K. Ploog, Phys. Rev. B 50, 18420 (1994).

${ }^{7}$ P. D. Wang, N. N. Ledentsov, C. M. Sotomayer Torres, P. S. Kop'ev, and V. M. Ustinov, Appl. Phys. Lett. 64, 1526 (1994).

${ }^{8}$ V. Bressler-Hill, A. Lorke, S. Varma, P. M. Petroff, K. Pond, and W. H. Weinberg, Phys. Rev. B 50, 8479 (1994).

${ }^{9}$ M. I. Alonso, M. Ilg, K. Ploog, and A. Trampert, J. Appl. Phys. 78, 1980 (1995).

${ }^{10}$ M. V. Belousov, N. N. Lendentsov, M. V. Maximov, P. D. Wang, I. N. Yasievich, N. N. Faleev, I. A. Kozin, V. M. Ustinov, P. S.
Kop'ev, and C. M. Sotomayer Torres, Phys. Rev. B 51, 14346 (1995).

${ }^{11}$ P. D. Wang, N. N. Ledentsov, C. M. Sotomayor Torres, I. N. Yassievich, A. Yu, Egovov, P. S. Kop'ev, and V. M. Ustinov, Phys. Rev. B 50, 1604 (1994).

${ }^{12}$ O. Brandt, H. Lage, and K. Ploog, Phys. Rev. B 43, 14285 (1991).

${ }^{13}$ T. C. Damen, K. Leo, J. Shah, and J. E. Cunningham, Appl. Phys. Lett. 58, 1902 (1991).

${ }^{14}$ J. Singh, K. K. Bajaj, and S. Chauduri, Appl. Phys. Lett. 44, 805 (1984); J. Singh and K. K. Bajaj, ibid. 48, 1077 (1986).

${ }^{15}$ J. Christen and D. Bimberg, Phys. Rev. B 42, 7213 (1990).

${ }^{16}$ R. Cingalani, O. Brandt, L. Tapfer, G. Scamarcio, G. C. La Rocca, and K. Ploog, Phys. Rev. B 42, 3209 (1990).

${ }^{17}$ Here, a 1-ML-high InAs island ( $\left.\delta=1 / \mathrm{ML}\right)$ distribution is assumed (Refs. 7-9); then the average coverage $p$ equals to $L_{z} / \mathrm{ML}$ for submonolayer structures. Note that if different values of the average coverage $p$ are taken into account to evaluate the standard deviation for different values of $L_{Z}$, the theoretical line will not vary significantly, but only become steeper, being more consistent with the experimental results. However, this treatment will have difficulties in the 1-ML, case where islands with a height of more than 1 ML must be considered to avoid $p=1$, in agreement with the experimental observations; see e.g., A. Patanè, A. Polimeni, M. Capizzi, and F. Nartelli, Phys. Rev. B 52, 2784 (1995).

${ }^{18}$ G. Bacher, C. Hartmann, H. Schmeizer, T. Held, G. Mahler, and 
H. Nickel, Phys. Rev. B 47, 9545 (1993).

${ }^{19}$ D. A. B. Miller, D. S. Chemla, D. J. Eilenberger, P. W. Smith, A. C. Gossard, and W. T. Tsang, Appl. Phys. Lett. 41, 679 (1982).

${ }^{20}$ H. Qiang, F. H. Pollak, C. M. Sotomayer Torres, W. Leitch, A. H. Kean, M. A. Straoscio, G. J. Iafrate, and K. W. Kim, Appl. Phys. Lett. 61, 1411 (1992).

${ }^{21}$ J. V. D. Veliadis, J. B. Khurgin, Y. J. Ding, A. G. Cui, and D. S. Katzer, Phys. Rev. B 50, 4463 (1994); Y. J. Chen, E. S. Koteles, J. Lee, J. Y. Chi, and B. S. Elman, Proc. SPIE 792, 168 (1987).

${ }^{22}$ D. Gammon, S. Rudin, T. L. Reinecke, D. S. Katzer, and C. S. Kyono, Phys. Rev. B 51, 16785 (1995).

${ }^{23}$ W. Z. Shen, W. G. Tang, S. C. Shen, S. M. Wang, and T. Anderson, Appl. Phys. Lett. 65, 2728 (1994).

${ }^{24}$ U. Bockelman and G. Bastard, Phys. Rev. B 42, 8947 (1990).

${ }^{25}$ H. Benisty, C. M. Sotomayer Torres, and C. Weisbuch, Phys. Rev. B 44, 10945 (1991).
${ }^{26}$ Z. Y. Xu, W. Ge, and Z. L. Yuan (unpublished).

${ }^{27}$ T. C. Damen, J. Shah, D. Y. Chemla, J. E. Cunningham, and J. M. Kuo, Phys. Rev. B 42, 7434 (1990).

${ }^{28}$ J. Feldmann, G. Peter, E. O. Göbel, P. Dawson, K. Moore, C. Foxon, and E. J. Elliot, Phys. Rev. Lett. 59, 2337 (1987); 60, 243 (1988).

${ }^{29}$ D. S. Citrin, Phys. Rev. Lett. 69, 3393 (1992).

${ }^{30}$ O. Brandt, G. C. La Rocca, A. Heberle, A. Ruiz, and K. Ploog, Phys. Rev. B 45, 3803 (1995).

${ }^{31}$ Z. Y. Xu, S. R. Jin, C. P. Luo, and J. Z. Xu, Solid State Commun. 87, 797 (1993).

${ }^{32}$ G. W. 't Hooft, W. A. J. A. van der Poel, L. W. Molenkamp, and C. T. Foxon, Phys. Rev. B 35, 8281 (1987).

${ }^{33}$ D. S. Citrin, Phys. Rev. B 47, 3832 (1993).

${ }^{34}$ L. C. Andreani, Solid State Commun. 77, 641 (1991). 